ARTICLE

Received 21 Mar 2013 | Accepted 13 Sep 2013 | Published 17 Oct 2013 DOI: 10.1038/ncomms3613

\title{
Molecular mechanism of strigolactone perception by DWARF14
}

Hidemitsu Nakamura ${ }^{1, \star}$, You-Lin Xue ${ }^{1, \star}$, Takuya Miyakawa ${ }^{1, \star}$, Feng Hou ${ }^{1}$, Hui-Min Qin ${ }^{1}$, Kosuke Fukui ${ }^{1}$, Xuan Shi ${ }^{1}$, Emi Ito ${ }^{2}$, Shinsaku Ito ${ }^{1}$, Seung-Hyun Park', Yumiko Miyauchi ${ }^{1}$, Atsuko Asano ${ }^{1}$, Naoya Totsuka ${ }^{1}$, Takashi Ueda ${ }^{2}$, Masaru Tanokura ${ }^{1} \&$ Tadao Asami ${ }^{1,3}$

Strigolactones (SLs) are phytohormones that inhibit shoot branching and function in the rhizospheric communication with symbiotic fungi and parasitic weeds. An $\alpha / \beta$-hydrolase protein, DWARF14 (D14), has been recognized to be an essential component of plant SL signalling, although its precise function remains unknown. Here we present the SL-dependent interaction of D14 with a gibberellin signalling repressor SLR1 and a possible mechanism of phytohormone perception in D14-mediated SL signalling. D14 functions as a cleavage enzyme of SLs, and the cleavage reaction induces the interaction with SLR1. The crystal structure of D14 shows that 5-hydroxy-3-methylbutenolide (D-OH), which is a reaction product of SLs, is trapped in the catalytic cavity of D14 to form an altered surface. The D14 residues recognizing $\mathrm{D}-\mathrm{OH}$ are critical for the SL-dependent D14 - SLR1 interaction. These results provide new insight into crosstalk between gibberellin and SL signalling pathways.

\footnotetext{
${ }^{1}$ Department of Applied Biological Chemistry, Graduate School of Agricultural and Life Sciences, The University of Tokyo, Tokyo 113-8657, Japan.

${ }^{2}$ Department of Biological Sciences, Graduate School of Science, The University of Tokyo, Tokyo 113-0033, Japan. ${ }^{3}$ JST, CREST, 4-1-8 Honcho, Kawaguchi, Saitama, 332-0012, Japan. * These authors contributed equally to this work. Correspondence and requests for materials should be addressed to T.A. (email: asami@mail.ecc.u-tokyo.ac.jp).
} 
T he terpenoid, small-compound strigolactones (SLs) are plant hormones that regulate plant shoot branching ${ }^{1,2}$, which is an important agronomic trait that determines crop yields. In addition, SLs stimulate the germination of root parasitic weeds $s^{3,4}$ that cause devastating crop damage ${ }^{5}$ and induce hyphal branching in symbiotic arbuscular mycorrhizal fungi that promote the growth of host plants by capturing essential inorganic nutrients from the soil ${ }^{6-8}$. Owing to the importance of SLs in agriculture, the mechanism and signal transduction pathway of SLs have been extensively researched to provide a perspective on the broader SL functions. However, the mechanisms responsible for SL recognition by plants are poorly understood.

SLs contain a structural core that consists of a tricyclic-lactone (ABC-ring) and a butenolide group (D-ring) that are connected via an enol ether linkage (compounds 1-4; Supplementary Fig. S1a). In plants, SLs are synthesized from carotenoids through carlactone (4) (ref. 9), a butenolide-containing compound with SL-like biological activity, by a sequential reaction of several enzymes, including an iron-binding protein D27 (ref. 9), the carotenoid cleavage dioxygenases 7 and 8, and a cytochrome P450 protein MAX1 (refs 10-12) (Supplementary Fig. S1b). In addition, studies on a set of branching mutants of some plant species have indicated that the $d 14$ and $d 3 /$ max2/rms 4 genes are closely related to a pathway downstream of SLs ${ }^{1,2,13,14}$ (Supplementary Fig. S1b).

$D 14$ genes encode $\alpha / \beta$-hydrolase family proteins ${ }^{13-16}$ and their mutants exhibit a highly branched phenotype and are insensitive to $\mathrm{SLs}^{13-14}$. The $d 3 /$ max $2 / \mathrm{rms}^{4}$ mutants are also SL insensitive ${ }^{1,2}$. D3/ MAX2/RMS4 genes encode members of the F-box-family protein ${ }^{17-}$ ${ }^{20}$ that participate in an Skp-cullin-F box (SCF) complex ${ }^{18}$ and have diverse roles in the plant lifecycle ${ }^{14,21-30}$. Recently, it has been reported that petunia DAD2, an orthologue of rice DWARF14 (D14) protein, can hydrolyse the enol ether linkage of GR24 and interacts with PhMAX2A, a petunia orthologue of Arabidopsis MAX2 protein, indicating that the formation of the DAD2PhMAX2A complex initiates an SCF-mediated signal transduction pathway ${ }^{31}$. However, it still remains unclear how the hydrolyzation of SLs induces the formation of the DAD2-PhMAX2 complex and what is/are the target(s) of the SCF complex.

The Arabidopsis gibberellin (GA) biosynthetic mutant ga1-3 exhibits enhanced shoot branching, and the overexpression of GA 2-oxidase genes in rice, in which GA levels are reduced, promotes tillering ${ }^{32}$. These results suggest that GAs regulate branching together with or without SLs. The DELLA-family proteins are key negative regulators of GA signalling through interaction with growth promoting transcription factors, such as PIFs. DELLA proteins have recently been reported to upregulate jasmonate (JA) signalling by physically interacting with JAZs, repressors of JA signalling ${ }^{33}$. Further, JAZs reportedly inhibit DELLAs - PIFs interactions. Thus, JA inhibits plant growth by inducing the degradation of JAZs and restoring the interaction of DELLAs with PIFs ${ }^{34}$ (Supplementary Fig. S1c). In addition, recent reports suggest that a transcription factor, which broadly regulates brassinosteroid signalling, BZR1, mediates growth response to GA via direct interaction with DELLAs ${ }^{35,36}$. These reports indicate that DELLAs have a role in regulating multiple signals.

To investigate whether DELLAs mediate crosstalk between GA and SL signalling pathways, we explored the interaction between the rice D14 and the DELLA protein SLR1. Here we show that the rice D14 interacts with SLR1 in an SL-dependent manner, thus contributing to the negative regulation of GA signalling ${ }^{37}$ (Supplementary Fig. S1d). Moreover, a series of crystallographic and biochemical studies suggest an advanced molecular mechanism of D14 in SL perception and signal transduction. Our results provide new insight into the crosstalk between the GA and SL signalling pathways.

\section{Results}

D14 interacts with the DELLA protein SLR1. To investigate whether D14 interacts with DELLA proteins, we performed a yeast two-hybrid ( $\mathrm{Y} 2 \mathrm{H})$ assay using D14 as the bait and SLR1, the only DELLA protein in rice, as the prey in the presence of various SLs, including $( \pm)-2^{\prime}$-epi-5-deoxystrigol (a racemic mixture of natural SLs $(+)-2^{\prime}-$ epi-5-deoxystrigol (1) and ( - )-ent-2'-epi-5deoxystrigol), ( \pm )-GR24 (a racemic mixture of $(+)$-GR24 (2)

a
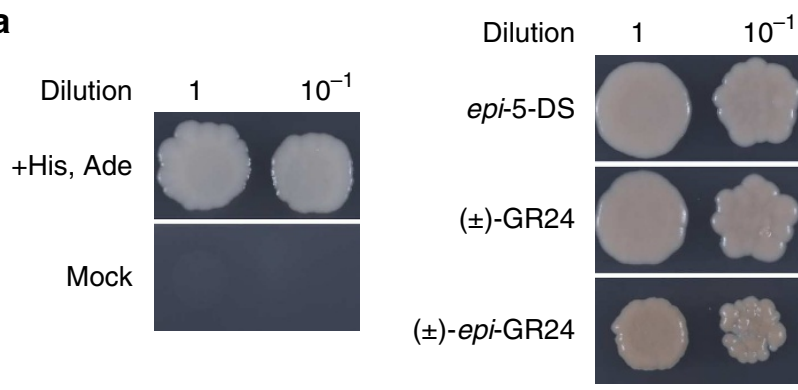

b

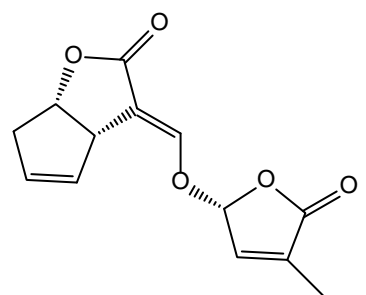

(-)-ent-2' -epi-GR7 (3)

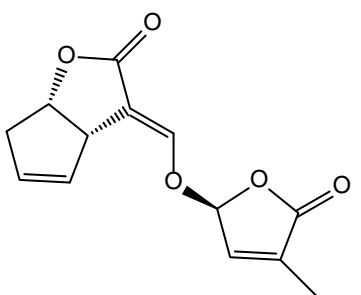

(-)-ent-GR7 (6)
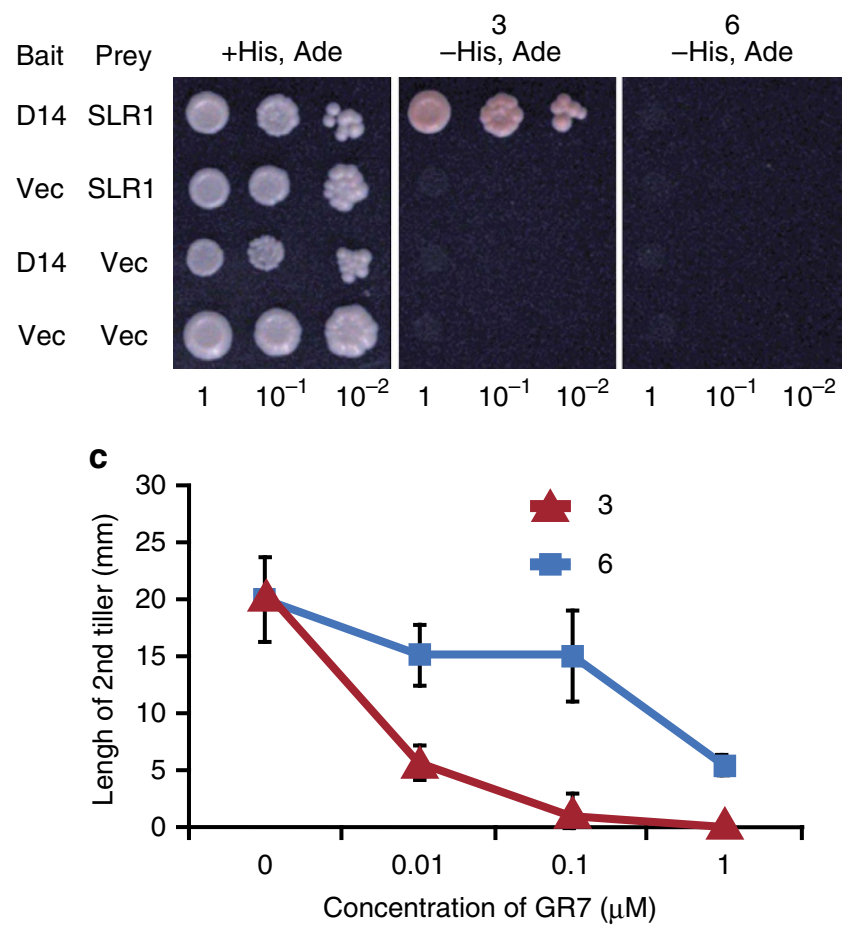

Figure 1 | Interaction between D14 and SLR1. (a) Growth of AH109 transformants with pGBK-D14 and pGAD-SLR1 on SD-His, Ade plates with various SLs. epi-5-DS: ( \pm )-2'-epi-5-deoxystrigol. ( \pm )-epi-GR24: ( \pm )-2'-epiGR24. (b) Structures of ( - )-ent-2'-epi-GR7 (3) and ( - )-ent-GR7 (6) and growth of $\mathrm{AH} 109$ transformants on an SD-His, Ade plate with or without $10 \mu \mathrm{M}(-)$-ent-2'-epi-GR7 (3) or ( - )-ent-GR7 (6). (c) Length of second tillers of 3-week-old rice treated with ( - )-ent-2'-epi-GR7 (3) or ( - )-entGR7 (6) (mean \pm s.d.; $n=5$ ). 

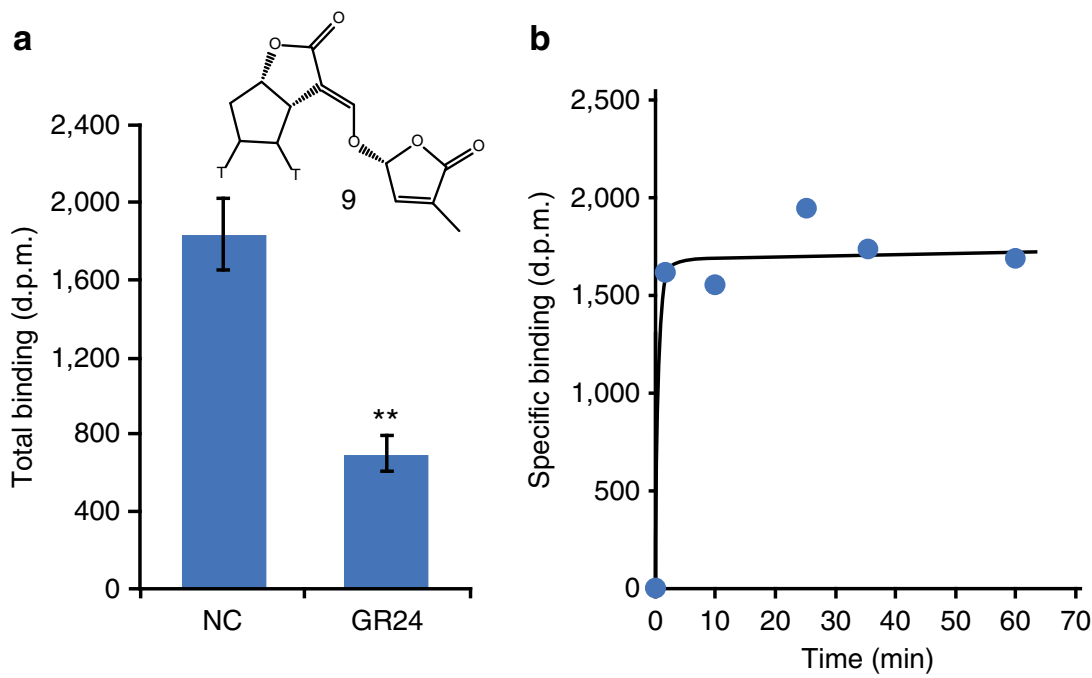

Figure 2 | SL-binding properties of D14 proteins. (a) Competition for $T_{2}-G R 7$ (9) binding to D14 by cold SLs (mean \pm s.d., $n=3$ ). NC, no competitor. d.p.m., disintegrations per minute. ${ }^{\star \star} P<0.01$ (Student's $t$-test) versus no competitor control. The structure of $T_{2}$-GR7 (9) is shown as an inset. (b) Association rates of $T_{2}-G R 7$ (9) and D14. The specific binding of $T_{2}-G R 7$ (9) reached one-half of the maximum within 1 min (mean; $n=2-3$ ).

and ( - )-ent-GR24 (5)), and ( \pm )-2'-epi-GR24 (a racemic mixture of $(+)-2^{\prime}$-epi-GR24 and ( - )-ent-2'-epi-GR24). The result indicated that these SLs induced a D14-SLR1 interaction (Fig. 1a). When ( - )-ent-GR7 (6) was added to the medium, this D14-SLR1 interaction was not observed, whereas when ( - )-ent2'-epi-GR7 (3), an enantiomer of ( - )-ent-GR7 (6), was added to the medium, this interaction was clearly observed (Fig. 1b). Among the other tested SLs, 5,6-dihydro-(-)-ent-2'-epi-GR7 (dihydro-GR7, 7; Supplementary Fig. S2b) also induced the interaction between D14 and SLR1, but 5,6,3', $4^{\prime}$-tetrahydro-( - )ent-2'-epi-GR7 (tetrahydro-GR7, 8; Supplementary Fig. S2b) did not induce the interaction (Supplementary Fig. S2a). The inhibitory effect of (-)-ent-2'-epi-GR7 (3) on the tiller bud outgrowth of rice was stronger than that of $(-)$-ent-GR7 (6) (Fig. 1c), indicating that the D14-SLR1 complex is formed in the presence of active SL forms. Similar results were obtained in the Y2H assay using dihydro-GR7 (7, active form) and tetrahydro-GR7 (8, less active form) (Supplementary Fig. S2). The direct interaction between D14 and SLs was determined using a non-equilibrium gel-permeation technique and $\left[5,6-{ }^{3} \mathrm{H}\right]-5,6-$ dihydro-(-)-ent-2'-epi-GR7 ( $\mathrm{T}_{2}$-GR7, 9; Fig. 2a), a radioactive SL. The results showed that D14 bound $\mathrm{T}_{2}$-GR7 (9) and that the binding was replaced in the presence of excess unlabelled ( \pm )-GR24 (Fig. 2a). The half-time for the association between D14 and $\mathrm{T}_{2}$-GR7 (9) was $<1 \mathrm{~min}$ (Fig. 2b), indicating that the binding reaction occurs rapidly. Such rapid receptor-ligand binding was also reported for the OsGID1-GA association ${ }^{38}$.

Subsequently, we performed a bimolecular fluorescence complementation (BiFC) analysis using chimeric proteins fused with split yellow fluorescent proteins (YFPs) to confirm whether the D14-SLR1 complex forms in vivo. Constructs for the expression of the fusion proteins, GFP-D14, nYFP-SLR1 and cYFP-D14, were transiently expressed in Arabidopsis protoplasts. The GFP-D14 fusion protein localized to the cytosol and the nucleus. A strong reconstituted YFP signal was observed demonstrating the nuclear interaction between D14 and SLR1 (Fig. 3a). This result is also consistent with the previous observations that DELLA proteins are localized to the nucleus ${ }^{39}$.

SLR1 has key roles as a negative regulator of GA signalling ${ }^{37}$ and interacts with OsGID1 in GA-dependent manner (Supplementary Fig. S1d). To confirm whether the SL-dependent
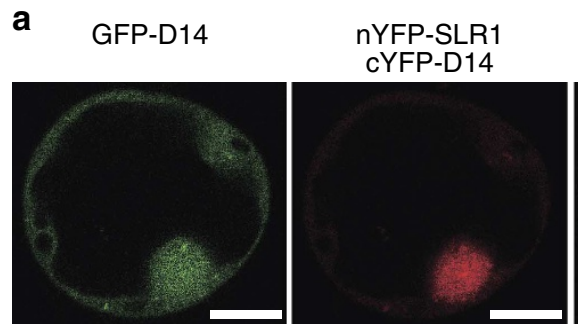

Merged

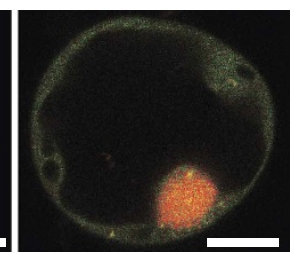

\section{b}

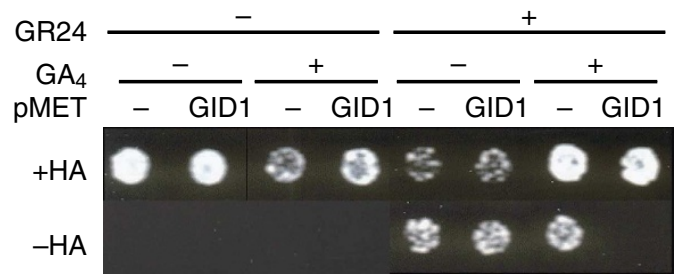

Figure 3 | D14-SLR1 interaction in planta and the competition between the D14-SLR1 interaction and the GID1-SLR1 interaction. (a) BiFC analysis of the D14-SLR1 interaction. D14-GFP, cYFP-D14 and nYFP-SLR1 were expressed transiently in the protoplasts of Arabidopsis suspension-cultured cells. Scale bars, $10 \mu \mathrm{m}$. (b) Growth of AH109 transformants with pBridgeBDD14-MGID1 (or pBridge-BDD14) and pGAD-SLR1 on SD-His, Ade, Met plates with or without $10 \mu \mathrm{M}( \pm)-G R 24$ and $1 \mu \mathrm{M} \mathrm{GA} 4$. When only ( \pm )GR24 was added to the growth medium, yeast colonies could grow independently of GID1 expression. In contrast, when both ( \pm )-GR24 and $\mathrm{GA}_{3}$ were added, the GID1-expressing yeast could not grow on the SD-His, Ade and Met medium.

D14-SLR1 interaction is competitive with the GA-dependent OsGID1-SLR1 interaction, we established a yeast three-hybrid (Y3H) system; D14 was used as the bait, SLR1 was used as the prey and OsGID1 was used as a D14 competitor (Fig. 3b). When both $\left( \pm\right.$ )-GR24 and $\mathrm{GA}_{4}$ were added, OsGID1-expressing yeast cells could not grow on the medium lacking L-histidine and adenine. This result indicates that the formation of the GR24-dependent D14-SLR1 complex is inhibited by the $\mathrm{GA}_{4}$-bound OsGID1, and the D14-interacting domain overlaps with the OsGID1-interacting domain in SLR1. 
D14 - SLR1 interaction requires enzymatic activity of D14. The enzymes belonging to the $\alpha / \beta$-hydrolase superfamily contain a nucleophile-histidine-acid catalytic triad, which is required to catalyse the hydrolytic cleavage of the substrates. The corresponding catalytic residues are conserved in D14 as Ser147 (nucleophile)-His297-Asp268 (Supplementary Fig. S3). To evaluate the D14 catalysis of the hydrolytic cleavage of SLs, we observed the degradation of $( \pm$ )-GR24 by D14 using an HPLC technique with a Chiralpak IA-3 column (Fig. 4a). Two peaks of ( - )-ent-GR24 (5) and (+)-GR24 (2) (peaks A and B, respectively, in Fig. 4a) were stably detected when $( \pm)-G R 24$ was incubated for $1 \mathrm{~h}$ without D14. In contrast, the peak of $(+)$ GR24 (2) disappeared when ( \pm )-GR24 was incubated with D14 for $1 \mathrm{~h}$, whereas the peak of $(-)$-ent-GR24 (5) was stable. The peak of ( - )-ent-GR24 (5) disappeared after overnight incubation with D14, indicating that ( - )-ent-GR24 (5) could be a substrate for the hydrolytic cleavage by D14, whereas (+)-GR24 (2) is a more favourable substrate than (-)-ent-GR24 (5). These results indicate that D14 has the catalytic ability to hydrolyse SLs, which is consistent with the observations of DAD2 (ref. 31), and, to some extent, this catalysis is stereoselective.

No interaction of DAD2 with PhMAX2A was observed by the mutation of the catalytic triad residues ${ }^{31}$. To evaluate the significance of the enzymatic reaction in the interaction between D14 and SLR1, we performed a mutational analysis of D14 using a $\mathrm{Y} 2 \mathrm{H}$ system. A mutation in the catalytic residue His297 (H297A) decreased the enzymatic activity of D14 towards GR24 (Fig. 4a) and abolished the SLR1 interaction in both the presence and absence of (-)-ent-2'-epi-GR7 (3) (Fig. 4b). In addition, the competitive binding assay using $\mathrm{T}_{2}$-GR7 (9) showed that the H297A mutant was capable to bind ( \pm )-GR24 (Fig. 4c). This result indicates that the catalytically inactive mutant could form a constitutive enzyme-substrate complex, which supports that the complex is not capable to interact with SLR1. Thus, the SL-dependent SLR1 interaction requires the enzymatic activity of D14 towards SLs.

Moreover, we examined whether the enzymatic cleavages of substrates are required for the interaction between D14 and SLR1. We prepared several SL analogues (compounds 10-13; Supplementary Fig. S4a), in which various moieties were linked to the D-ring by ether or ester bonds. 3,5-dimethoxybenzoyloxy D-ring (12) and anthracene-9-carbonyloxy D-ring (13) induced $\mathrm{D} 14-\mathrm{SLR} 1$ interaction a concentration of $10 \mu \mathrm{M}$ in the $\mathrm{Y} 2 \mathrm{H}$ assay, although methoxy D-ring and acetoxy D-ring did not induce D14-SLR1 at this concentration. However, acetoxy D-ring (11) induced D14 - SLR1 interaction at a high concentration $(100 \mu \mathrm{M})$, whereas methoxy D-ring (10) did not (Supplementary Fig. S4b). These data correspond to the results of the bioassay on the tiller bud outgrowth (Supplementary Fig. S4c). The fact that all active chemicals possessed an ester bond and that methoxy D-ring was inactive in the $\mathrm{Y} 2 \mathrm{H}$ and the tillering inhibition assay suggests that the enzymatic cleavage of substrates by D14 is important for the interaction between D14 and SLR1, and the ester link is susceptible to hydrolysis by D14. Consistent with this hypothesis, we observed the D14 catalysis of the hydrolytic cleavage of anthracene-9-carbonyloxy D-ring using an HPLC technique (Supplementary Fig. S4d).

D14 forms a complex with D-OH to mediate SLR1 interaction. On examination of the recent model of D14 activation, it is
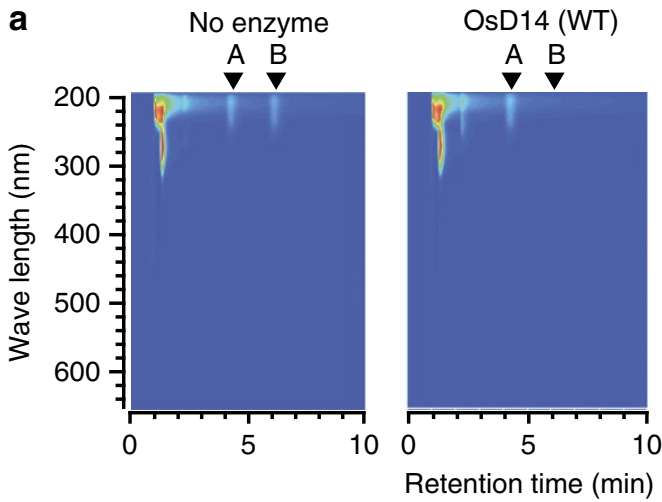

A

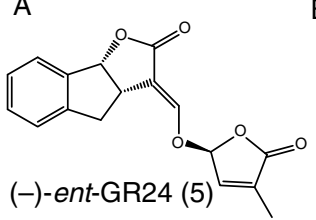

B<smiles>CC1=CC(COC2=C3Cc4ccccc4C3OC2=O)OC1=O</smiles>

b

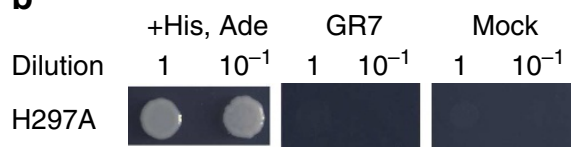

OsD14 (H297A)
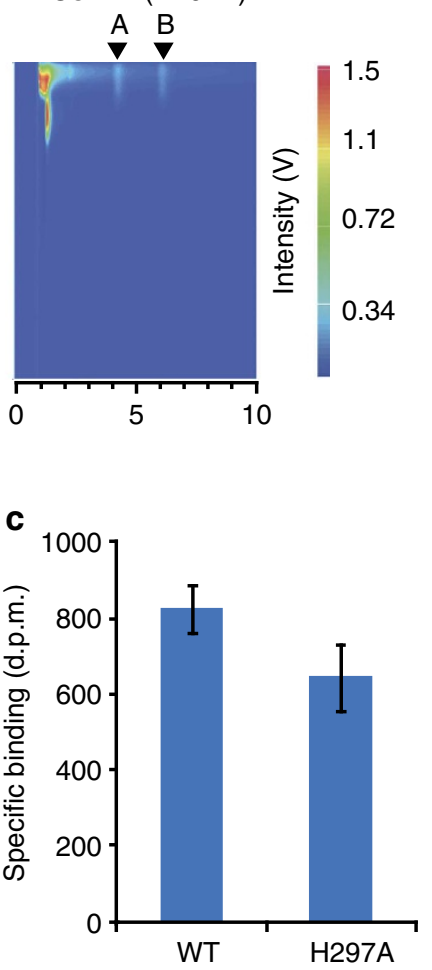

Figure 4 | Catalytic activity of D14 in the interaction with SLR1. (a) Enzymatic degradation of ( \pm )-GR24 by D14. ( \pm )-GR24 (10 $\mu$ M) was incubated with or without purified D14 for $1 \mathrm{~h}$ at $37^{\circ} \mathrm{C}$. The reaction solutions were extracted with ethyl acetate, and the organic layer was subjected to HPLC analyses using a Chiralpak IA-3 column. Peaks A and B were confirmed to represent ( - )-ent-GR24 (5) and (+)-GR24 (2), respectively, as described in the Methods section. The experiments were repeated three times with similar results. (b) Growth of AH109 transformants with pGAD-SLR1 and the wild-type or mutated D14 cDNA on pGBK-T7 on an SD-His, Ade plate with or without $10 \mu \mathrm{M}(-$ )-ent-2'-epi-GR7 (3). (c) Comparison of the specific binding of T2-GR7 (9) to wild-type D14 and to H297A mutant D14 (mean \pm s.d., $n=3$ ). 
predicted that the formation of a reaction intermediate may induce structural changes that facilitate the interaction between D14 and its target proteins ${ }^{31,40}$. The crystal structure of D14 with a GR24-degradation intermediate, 2,4,4-trihydroxy-3-methyl-3butenal, could propose the reaction mechanism for producing a hydroxy $\mathrm{ABC}$-ring $(\mathrm{ABC}-\mathrm{OH})$ and a hydroxy D-ring $(\mathrm{D}-\mathrm{OH})$ from GR24 (ref. 41), although it remains unclear whether the intermediate complex functions as an active form of SL signalling and no significant structural changes are found between the overall structures of apo and the intermediate. When we tried to co-crystallize D14 with several SLs including $( \pm)$-GR24 and (-)-ent-2'-epi-GR7 (3) and to soak the substrates into D14 crystals, we solved the crystal structure of D14 complexed with a reaction product $\mathrm{D}-\mathrm{OH}$ at a $2.10-\AA$ resolution (Fig. $5 \mathrm{a}$ ). In this experiment, the cavity of D14 in the crystal could be exposed to solvent (Supplementary Fig. S5a,b) and, therefore, a substrate and a reactive water molecule could access to the cavity even in the crystal. D-OH may be produced via the reported intermediate, 2,4,4-trihydroxy-3-methyl-3-butenal ${ }^{41}$ from the soaked (-)-ent-2'-epi-GR7 (3) by an in-crystal activity of D14. This product is located at a site far from the catalytic triad residues and is surrounded by Val194, Ser270 and several aromatic residues, such as Phe186, Trp205, Tyr209 and Phe245 (Fig. 5b). These residues make favourable hydrophobic and/or van der Waals interactions with $\mathrm{D}-\mathrm{OH}$. In addition, the indole amine of Trp205 forms a hydrogen bond with the oxygen atom of the $\mathrm{C} 2^{\prime}$ hydroxy group, which could arise from the catalytic reaction of D14, instead of a water molecule in the structure of apo D14 (Supplementary Fig. S5). In the complex structure, $\mathrm{D}-\mathrm{OH}$ functions as a plug for the catalytic cavity of D14 and induces a hydrophilic patch in an overall hydrophobic surface between the two $\alpha$-helices ( $\alpha \mathrm{A}$ and $\alpha \mathrm{B})$ in the cap structure of D14 (Fig. 5c).

Large structural differences were not observed between the apo and D-OH-bound D14 molecules, although the side chain of Phe 245 was moved $1.3 \AA$ towards D-OH (Fig. 5d). In addition, the $\mathrm{C} \alpha$ atoms of the $\alpha \mathrm{A}-$ and $\alpha \mathrm{D}$-helices and the large loop connecting to the $\alpha \mathrm{A}$-helix were changed with the root mean square distance of $>0.4 \AA$ upon D-OH binding (Supplementary Fig. S6). The Phe 245 residue located on the $\alpha$ D-helix makes contact with the $\alpha \mathrm{A}$-helix and the loop. Therefore, these regions seem to be directly affected by the side-chain rearrangement of
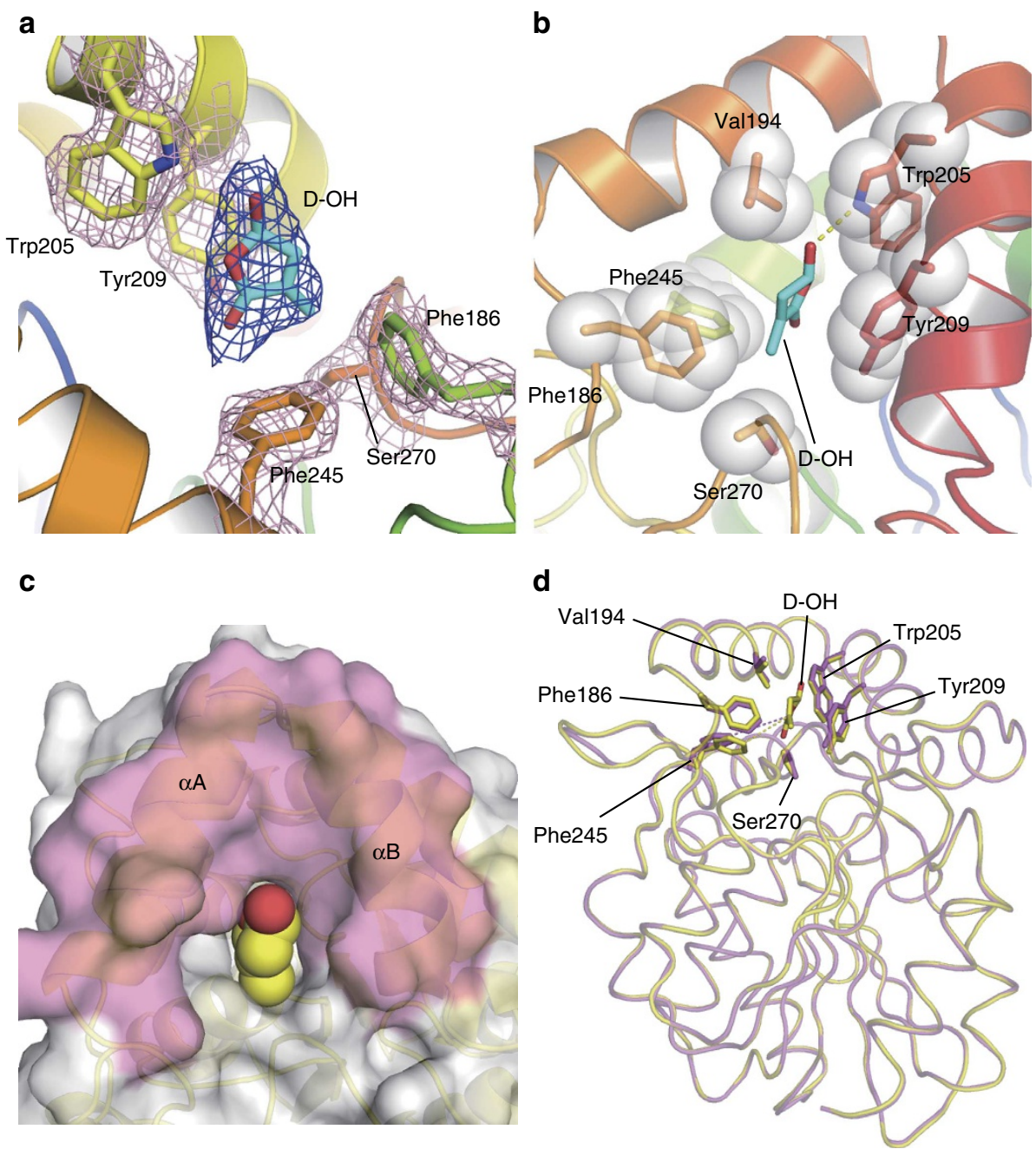

Figure 5 | D-OH recognition by D14. (a) Side view of D-OH bound to D14. D-OH and the surrounding residues are shown as a stick model along with the $2 F_{\mathrm{o}}-F_{\mathrm{c}}$ electron density coloured in blue and purple, respectively. (b) Binding site of $\mathrm{D}-\mathrm{OH}$. The residues surrounding $\mathrm{D}-\mathrm{OH}$ are represented by a stick-and-sphere model. The dashed line shows a hydrogen bond between D-OH and the indole amine of Trp205. (c) Plug-like binding of D-OH to the catalytic cavity of D14. D-OH is shown using a sphere representation. (d) Superposed structure of apo and D-OH-bound D14. The crystal structures of apo and D-OH-bound D14 are shown as pink and yellow ribbon diagrams, respectively. D-OH and its surrounding residues are represented by stick diagrams. Phe245 is the only residue that moves $(1.3 \AA)$ towards $\mathrm{D}-\mathrm{OH}$. 
a

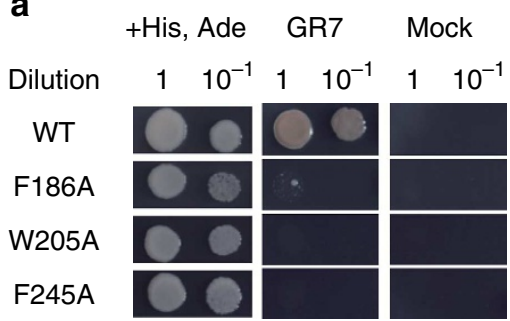

b

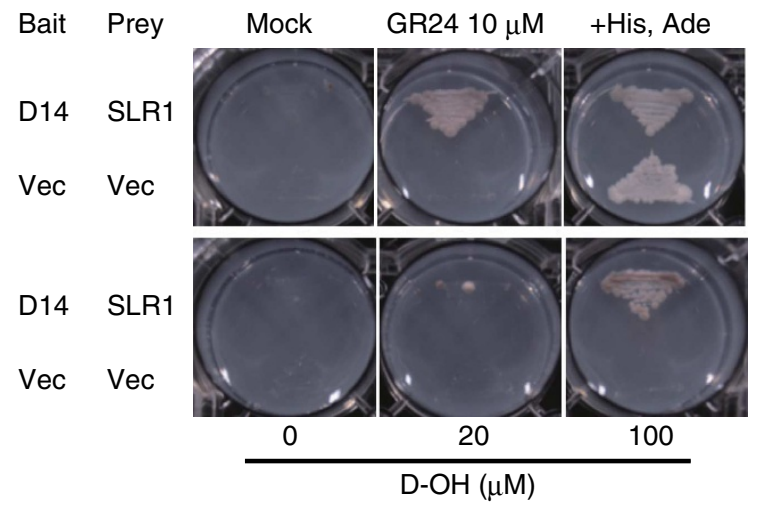

C

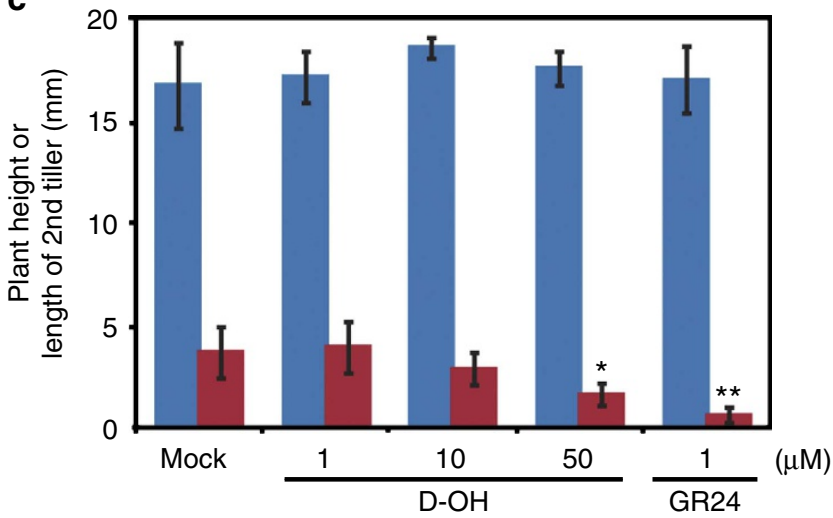

Figure 6 | Effects of D-OH in SLR1 interaction and in the outgrowth of tiller buds. (a) Growth of AH109 transformants with pGAD-SLR1 and the wild-type or mutated D14 CDNA on pGBK-T7 on an SD-His, Ade plate with or without $10 \mu \mathrm{M}$ ( - )-ent-2'-epi-GR7 (3). (b) Growth of AH109 transformants on an SD-His, Ade plate with or without D-OH. (c) Inhibition of the growth of second tiller buds by $\mathrm{D}-\mathrm{OH}$. The plant height (blue) and length of the second tillers (red) of 3-week-old rice plants that were treated with $\mathrm{D}-\mathrm{OH}$ or $\left( \pm\right.$ )-GR24 (mean \pm s.d.; $n=6$ ) are shown. ${ }^{*} P<0.05$ and ${ }^{\star \star} P<0.01$ (Student's $t$-test) versus non-treated control.

Phe245. In the previous study, it has been shown that the D14 orthologue DAD2 is destabilized in the reaction process of GR24 (ref. 31). The trypsin digestion assay showed that the D14 protein (wild type) was efficiently degraded when $( \pm)$-GR24 or $(-)$-ent2 '-epi-GR7 (3) was added in the reaction solution (Supplementary Fig. S7). On the other hand, the degradation of the catalytically inactive $\mathrm{H} 297 \mathrm{~A}$ mutant was not affected by adding ( \pm )-GR24 or ( - )-ent-2'-epi-GR7 (3). These results are consistent with structural changes induced by $\mathrm{D}-\mathrm{OH}$ binding.

To evaluate the significance of $\mathrm{D}-\mathrm{OH}$ binding in the interaction with SLR1, we performed a mutational analysis of D14 using a Y2H system. The SLR1 interactions in a GR7-dependent manner were lost when Phe186, Trp205 or Phe245 were mutated to alanine (Fig. 6a). These residues contact $\mathrm{D}-\mathrm{OH}$ and/or form the
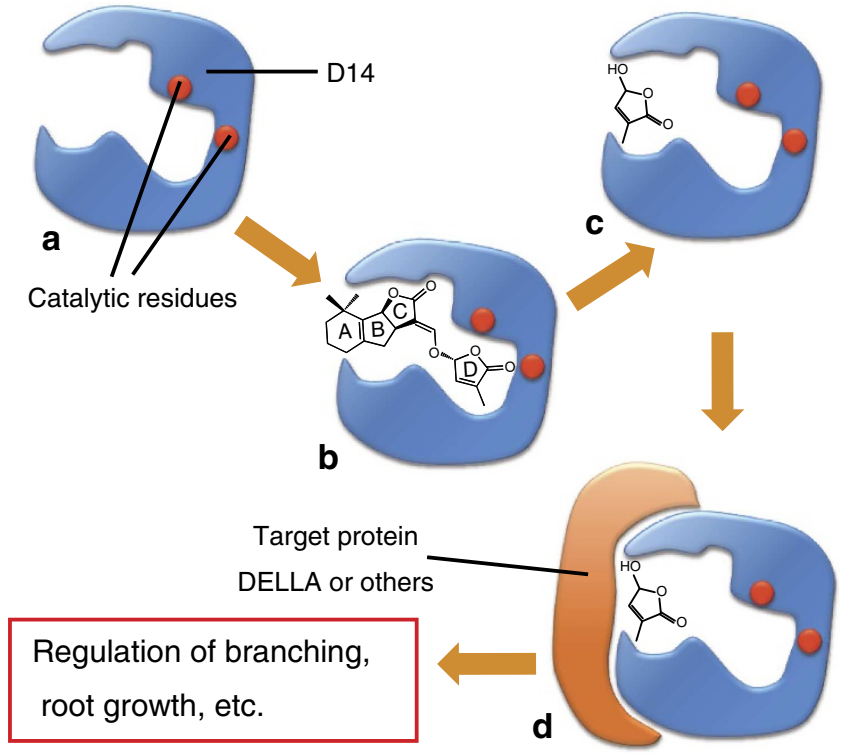

Figure 7 | A model of SL recognition and SL hydrolysation and the regulation of SL-induced signal transduction by D14. (a) The empty form of D14. (b) SL-bound D14. (c) SL is catabolized by D14, and D-OH is produced. $\mathrm{D}-\mathrm{OH}$ then remains at the aperture area of the binding cavity of D14. (d) The hydrophobic surface of the cap structure of D14 is recognized by a target protein, such as SLR1, and the D14-SL-target protein complex modulates downstream signalling. (+)-5-deoxystrigol was shown in this model as a representative of natural SLs.

hydrophobic surface observed on the cap structure. In addition, the side chain of Phe 245 is rearranged on $\mathrm{D}-\mathrm{OH}$ binding to slightly modify the local structure of the contact region with the residue, especially the $\alpha \mathrm{A}$-helix. The enzymatic activity towards GR24 was also decreased by the mutation of Trp205 as well as by the mutation of the catalytic residue, His297 (Supplementary Fig. S8). As the interaction between D14 and SLR1 requires an enzymatic activity, the functional contribution of $\mathrm{D}-\mathrm{OH}$ towards the D14 - SLR1 interaction is unclear in these mutations. Trp205 may be essential to the binding of the GR24 molecule as well as the D-OH molecule in the cavity. In contrast, the F245A mutant showed a slightly lower enzymatic activity towards GR24 compared with that of wild-type D14 (Supplementary Fig. S8), raising the possibility that the $\mathrm{D} 14-\mathrm{D}-\mathrm{OH}$ complex mediates the interaction with SLR1 in which the $\mathrm{D}-\mathrm{OH}$-induced surface and/or structural change is crucial. Consistent with the importance of $\mathrm{D}-\mathrm{OH}$ in the D14 - SLR1 interaction, the addition of a high concentration of $\mathrm{D}-\mathrm{OH}$ induced the interaction between $\mathrm{D} 14$ and SLR1 in the $\mathrm{Y} 2 \mathrm{H}$ assay (Fig. 6b) and significantly suppressed the outgrowth of the tiller bud of a rice $d 27-1$ mutant without affecting the plant height (Fig. 6c), indicating that the inhibition of the tiller outgrowth was not due to the toxic effect of a high concentration of $\mathrm{D}-\mathrm{OH}$. On the contrary, the addition of a high concentration of $\mathrm{D}-\mathrm{OH}$ did not inhibit the outgrowth of the tiller bud of the SL-insensitive $d 3$-1mutant rice (Supplementary Fig. S9). In agreement with the previous study ${ }^{42}$, the addition of a low concentration of $\mathrm{D}-\mathrm{OH}(\sim 10 \mu \mathrm{M})$ did not inhibit outgrowth of the tiller bud of rice and did not induce D14 - SLR1 interaction in the $\mathrm{Y} 2 \mathrm{H}$ assay. These results support that a reaction product D-OH induces the interaction between D14 and SLR1 as a mature form of SL to transmit the SL signalling (Fig. 7).

\section{Discussion}

Until now, D14 proteins were known to hydrolyse the enol ether linkage of SLs to produce $\mathrm{ABC}-\mathrm{OH}$ and $\mathrm{D}-\mathrm{OH}^{41}$ and function in 
SL signalling ${ }^{31}$, but the mechanism for how SL perception is converted to SL signal transduction has not been clarified. Our results raise the possibility that $\mathrm{D}-\mathrm{OH}$ induces the D14-SLR1 interaction and acts as an active branching inhibitor. In SLs and in compounds with SL-like biological activities, the D-ring unit and the enol ether section $(>\mathrm{C}=$ CH-O-) are common structures, whereas other moieties are rather flexible (Supplementary Fig. 1a). In addition, active SLs are catabolized by $\mathrm{D} 14$ and a reaction product $\mathrm{D}-\mathrm{OH}$ is trapped in the cavity of D14. On the basis of these findings, we propose an advanced molecular mechanism of D14 in which D-OH functions as a mature form of SL to transmit the SL signalling (Fig. 7): D14 functions in the conversion of SLs to the bioactive form D-OH and participates in SL signalling as a complex with $\mathrm{D}-\mathrm{OH}$ by interacting with the target proteins such as SLR1. The addition of $\mathrm{D}-\mathrm{OH}$ alone at the same concentration as the addition of other active SLs did not induce a D14-SLR1 interaction in the Y2H assay (Fig. 6b) and did not inhibit the tillering of the $d 27-1$ mutant (Fig. 6c). Although further structural and biochemical experiments are needed, proper localization of $\mathrm{D}-\mathrm{OH}$ in the aperture of the binding cavity of D14 may require the prior binding of SL followed by hydrolytic cleavage to produce $\mathrm{D}-\mathrm{OH}$. Alternatively, D-OH may not be transported and/or incorporated efficiently into the nuclei in plants.

GA is received by the GA receptor GID1, which promotes the formation of the GA-GID1-DELLA protein complex. This complex is then recognized by the $\mathrm{SCF}^{\mathrm{SLY} 1 / \mathrm{GID} 2}$ complex, which recruits DELLA proteins for proteasomal degradation ${ }^{33}$ (Supplementary Fig. 1c). As GID1 and D14 are members of the $\alpha / \beta$-hydrolase family and SLY1/GID2 and MAX2/D3 are members of the F-box family, D14 and MAX2/D3 are assumed to function in SL signalling in a manner similar to that of GID1 and SLY1/GID2 in GA signalling, and our results suggest that DELLA proteins are potential candidates for the target of the D14-MAX2/D3 complex (Fig. 7). Direct interactions of DELLA proteins with other hormone signalling factors, such as JAZs and BZR1, have recently been reported to mediate the crosstalk between GA and JA signalling ${ }^{33,34}$, and between GA and brassinosteroid signalling ${ }^{35,36}$, respectively, indicating a pivotal role of DELLAs in regulating multiple signals. Our results suggest that the D14 - DELLA interaction mediates the crosstalk between GA and SL signalling pathways. However, although both SL and GA repress the elongation of tillering buds, each hormone has distinct functions. For example, it is well known that the treatment of GA results in elongation of shoots, although the SL treatment does not cause the shoot elongation. Identification of factors involved in the D14-DELLA complex, such as PIFs in the JAZ-DELLA interaction, under various environmental and developmental situations will provide a better understanding of the mechanisms for the commonality and difference of actions of these hormones in response to environmental and plant developmental processes.

Our model of the SL perception by D14 explains why a wide variety of natural SLs and analogues can exert their activity as branching inhibitors; the D-ring moiety of SLs is essential for hormonal activity ${ }^{42}$. Although SLs also function in arbuscular mycorrhizal fungi hyphal branching and in seed germination of parasitic plants, structure-activity relationships are different among individual organisms $\mathrm{s}^{4,8,42}$, suggesting that each organism uses a distinct SL recognition system. The identification of SL perception mechanisms in different organisms would provide further information. Taken together, our findings contribute to the development of novel SL analogues and SL-signalling inhibitors for unveiling further details of SL signalling and for controlling plant growth and protecting crops from parasitic weeds to increase crop yields.

\section{Methods}

Plant materials and growth conditions. An SL-deficient $d 10-1$ and $d 27-1$ mutants of the Japonica-type rice cultivar (Oryza sativa L. cv. Shiokari) was used in this study. Rice plants were grown as described previously ${ }^{43}$ with some modifications. Germinated seeds were transferred into hydroponic culture medium ${ }^{2}$ that was solidified with $0.6 \%$ agar and cultured at $25^{\circ} \mathrm{C}$ under fluorescent white light with a 16-h light/8-h dark photoperiod for 6 days. Each seedling was transferred to a glass vial containing $12 \mathrm{ml}$ of a sterilized hydroponic culture solution with or without chemicals and grown under the same conditions for 6 days.

Plasmid construction. All of the PCR primers used for plasmid construction are listed in Supplementary Table S1. For the Y2H assay, pGADT7 (Clontech) and pGBK-T7 (Clontech) were used as the expression vectors. The D14 open reading frame fragment was amplified from total complementary DNA from rice seedlings by standard PCR with the D14-5-EcoRI and D14-3-BamHI primers and cloned into the EcoRI-BamHI site of the pGBK-T7 vector to construct pGBK-D14. Sitedirected mutagenesis was performed by using the PrimeSTAR mutagenesis basal kit (Takara Bio Inc.) with pGBK-D14 as a template and the following primer sets:

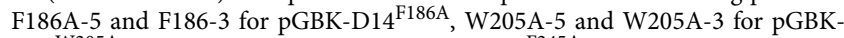
D14 ${ }^{\mathrm{W} 205 \mathrm{~A}}, \mathrm{~F} 245 \mathrm{~A}-5$ and F245A-3 for pGBK-D14 ${ }^{\mathrm{F} 245 \mathrm{~A}}$, and H297A-5 and H297A3 for pGBK-D14 $\mathrm{H} 297 \mathrm{~A}$. The SLR1 open reading frame fragment was amplified with the SLR1f and SLR1r primers and cloned into the NdeI-EcoRI site of the pGADT7 vector to construct pGAD-SLR1. To prepare the purified D14 proteins, the D14 cDNA fragment (residues $54-318$ ) from rice was inserted into the pET-49b expression vector (Novagen). The fusion proteins contained a GST-tag at the amino terminus and an HRV3C protease site between GST and D14. To construct vectors for the $\mathrm{BiFC}$ assay, the $\mathrm{N}$-terminal $(1-154$ a.a.) and carboxy-terminal $(155-239$ a.a.) fragments of enhanced YFP (eYFP; Clontech) were amplified by standard PCR using the following primer sets: eYFP-N0-f and eYFP-N0-r for eYFP 1-154 (N0 fragment) and eYFP-MC-f and eYFP-MC-r for eYFP 155-238 with methionine at its $\mathrm{N}$ terminus (MC fragment). The N0 and MC fragments were inserted into pUGW0 (ref. 44) at Aor51HI sites to produce nYFP/pUGW0 and cYFP/pUGW0, respectively. To construct entry clones, the D14 and SLR1 fragments were amplified by two-step adaptor PCR as described by the manufacturer (Invitrogen). The templates were pGBK-D14 and pGAD-SLR1, and the following primer sets were used for the first PCR reaction: attB1-D14 and attB2-D14 for D14, and attB1-SLR1 and attB2-SLR1 for SLR1. The second PCR was performed with the attB1 and attB2 adaptor primers, and the amplified product was then inserted into pDONR221 via a BP reaction, as described by the manufacturer (Invitrogen) to construct pENTR-D14 and pENTR-SLR1. The DNA fragments from pENTR-D14 and pENTR-SLR1 were transferred into cYFP/ pUGW0 and nYFP/pUGW0, respectively, via an LR reaction as described by the manufacturer (Invitrogen).

Y2H and Y3H assays. The Matchmaker Two-Hybrid System (Clontech) was used for the Y2H assay. pGBK-D14 served as the bait and pGAD-SLR1 served as the prey. Saccharomyces cerevisiae AH109 strains that were transformed with the bait and prey plasmids were grown in liquid media for 2 days, and the cell cultures were adjusted to $\mathrm{OD}_{600}=10$. Plate assays (synthetic defined (SD)-His, Ade) were performed according to the manufacturer's protocol, with the modification that the plate media either did or did not contain SLs. For the $\mathrm{Y} 3 \mathrm{H}$ assay, the yeast strains AH109 and Y187 and the plasmids pGADT7 and pBridge were obtained from Takara Bio Inc. pBridge-BDD14-MGID1 was constructed by fusing D14 cDNA with the GAL4-BD domain and inserting GID1 cDNA into the site downstream of pMET1. Strain Y187 was transformed with the pBridge vector harbouring BDD14 and pMET::GID1 or BDGID1 alone and selected on SD media lacking L-tryptophan (SD-Trp). Strain AH109, harbouring pGADT7-SLR1, grew on SD media lacking L-leucine and L-methionine (SD-Leu, Met). By mating both strains, the transformants carrying both plasmids (pBridge and pGADT7-SLR1) were selected on SD-Trp, Leu and Met. For the assay, the transformants were incubated on SD-Trp, Leu and Met media that lacked adenine hemisulphate and L-histidine but contained 3-aminotriazole.

SL-binding assay. For the SL-binding assay, $\left[5,6-{ }^{3} \mathrm{H}\right]-5,6$-dihydro- $(-)$-ent- $2^{\prime}$-epi GR7 $\left(T_{2}\right.$-GR7, 9) was used as the radioactively labelled GR7. This labelled GR7 was synthesized with the help of Institute of Isotopes Co., Ltd (Budapest, Hungary). In vitro assays for SL binding were performed according to the method for the GA-binding assay ${ }^{38}$ with some modifications. A total of $2 \mu \mathrm{g}$ of the purified OsD14 protein was added to $300 \mu \mathrm{l}$ of PBS buffer, and each sample was incubated with $100 \mu \mathrm{l}$ of $\mathrm{T}_{2}$-GR7 $(9 ; 1.13 \mathrm{nmol})$ in the presence of 442 -fold excess of unlabelled $( \pm)-G R 24(500 \mu \mathrm{M})$ for nonspecific binding or in the absence of excess unlabelled GR24 for total binding. Afterwards, $100 \mu \mathrm{l}$ of the mixture was fractionated on a NAP-5 column (GE Healthcare). After discarding a void volume of PBS eluate $(600 \mu \mathrm{l})$, a $200-\mu \mathrm{l}$ fraction was collected, and its radioactivity was measured. The specific binding activity was calculated by subtracting the nonspecific binding from the total binding.

BiFC assay. For the BiFC assay, an Arabidopsis suspension culture was transiently transformed according to methods that were described previously ${ }^{45}$. The 
transformed cells were observed with a confocal laser microscopes. Multicolour observations were performed with an LSM710 confocal microscope (Carl Zeiss), as described previously ${ }^{46,47}$. A thaliana-cultured cells were placed on eight-well multitest glass slides (MP Biomedicals) and covered with a 0.12-0.17-mm-thick coverslip $(24 \times 60 \mathrm{~mm}$; Matsunami). The acquired images were analysed with the IMAGE J software program (National Institutes of Health).

Protein preparation. Rosetta (DE3) cells (Novagen) transformed with the expression vector for D14 were grown in Luria-Bertani broth at $37^{\circ} \mathrm{C}$ to an $\mathrm{OD}_{600}$ of $\sim 0.6$ and induced with $0.2 \mathrm{mM}$ isopropylthio- $\beta$-galactoside at $25^{\circ} \mathrm{C}$ for $16 \mathrm{~h}$. The cells were collected and resuspended in extraction buffer $(20 \mathrm{mM}$ Tris- $\mathrm{HCl}$ (pH 8.5), $500 \mathrm{mM} \mathrm{NaCl}$ and $1 \mathrm{mM}$ dithiothreitol (DTT)), which included $10 \%$ glycerol for D14. After sonication on ice, the lysate was centrifuged at 40,000 $\mathrm{g}$ in an Avanti J-E rotor (Beckman Coulter) for $30 \mathrm{~min}$ at $4{ }^{\circ} \mathrm{C}$, and the supernatant was loaded onto a disposable polypropylene column packed with glutathione Sepharose $4 \mathrm{~B}$ resin (GE Healthcare). The column was washed with the extraction buffer. Oncolumn cleavage was performed overnight at $4{ }^{\circ} \mathrm{C}$ by adding HRV3C protease, and the target protein was eluted with each elution buffer as follows: $20 \mathrm{mM}$ MES$\mathrm{NaOH}$ (pH 6.5), $150 \mathrm{mM} \mathrm{NaCl}, 10 \%$ glycerol and $1 \mathrm{mM}$ DTT. After the eluted product was diluted fourfold in elution buffer lacking $\mathrm{NaCl}$, D14 was further purified using a Resource S column (GE Healthcare). D14 was eluted from a column with a $75-500-\mathrm{mM} \mathrm{NaCl}$ gradient in $20 \mathrm{mM}$ MES- $\mathrm{NaOH}$ (pH 6.5), $10 \%$ glycerol and $1 \mathrm{mM}$ DTT.

Crystallization. The purified protein was concentrated to $6.5 \mathrm{mg} \mathrm{ml}^{-1}$ in buffer containing $20 \mathrm{mM}$ MES-NaOH (pH 6.5) and $0.5 \mathrm{M}$ arginine hydrochloride. The D14 crystals were grown in sitting drops at $4{ }^{\circ} \mathrm{C}$ by vapour diffusion. The reservoir solution contained 0.1 M HEPES (pH 7.5), 8\% PEG20000, and 2\% 1,4-dioxane. Drops were prepared by mixing $0.8 \mu \mathrm{l}$ of purified protein with $0.8 \mu \mathrm{l}$ of reservoir solution. Crystals of $\sim 100 \mu \mathrm{m}$ in length appeared within 3 days. The crystals were soaked in the reservoir solution containing $0.2 \mathrm{M}(-)$-ent-2'-epi-GR7 (3) to obtain the protein-ligand complex.

Data collection and structure determination. The crystals were transferred into a reservoir solution containing $22 \%$ ethylene glycol before they were flash frozen in liquid nitrogen. X-ray diffraction data from a D14 crystal were collected on the AR-NE3A beamline at Photon Factory (Tsukuba, Japan). For a crystal of the protein-ligand complex, a diffraction data set was collected using an in-house $\mathrm{X}$-ray diffractometer (Rigaku FR-E rotating-anode X-ray generator with R-AXIS VII imaging-plate detector). The diffraction images were integrated and scaled using the XDS package ${ }^{48}$. Molecular replacement was performed by using the CCP4 program Molrep with the Bacillus subtilis stress-response regulator RsbQ (PDB: $1 \mathrm{WOM})^{49}$ as the initial model. $\operatorname{Coot}^{50}$ was used to manually fit the protein models. Model refinement was performed with the CCP4 program Refamc5 ${ }^{51}$. Most of the residues in the refined structures were in the most favourable region of the Ramachandran plot, and no residue was in the disallowed resign based on PROCHECK $^{52}$ (D14: residues in most favourable region, $97.1 \%$ and residues in allowed region, $2.9 \%$; D14-D-OH complex: residues in most favourable region, $97.0 \%$ and residues in allowed region, $3.0 \%$ ). The statistical analyses of the data sets and refined structures are listed in Table 1.

Enzymatic in vitro assays. The D14 in vitro assays were performed in a total volume of $1 \mathrm{ml}$ of PBS buffer containing $10 \mu \mathrm{M}( \pm)$-GR24. Purified D14 was added at a final concentration of $12 \mu \mathrm{g} \mathrm{ml}^{-1}$ and incubated for $16 \mathrm{~h}$ at $37^{\circ} \mathrm{C}$. Next, $100 \mathrm{mg}$ of $\mathrm{NaCl}$ was added to each reaction solution, and the reaction solutions were extracted with $400 \mu \mathrm{l}$ of ethyl acetate three times. The organic layers were combined and dried in vacuo and dissolved in $15 \mu \mathrm{l}$ of ethyl acetate. For each layer, $10 \mu \mathrm{l}$ was applied to the HPLC analyses. The normal-phase chromatographic separation was performed on a Jasco HPLC system that was equipped with an HPLC pump of model PU 1580 (Jasco) and a photodiode array detector MD1505 (Jasco), and was controlled by the ChromNAV (Ver. 1.16.02) software program (Jasco). The analytical column was a Chiralpak IA-3 column $(\Phi 2.1 \times 150 \mathrm{~mm}$, Daicel) or a Chiralpak AD column $(\Phi 2.1 \times 150 \mathrm{~mm}$, Daicel). The analytes were eluted under isocratic conditions using $85 \% n$-hexane and $15 \%$ ethanol at a flow rate of $0.5 \mathrm{ml} \mathrm{min}^{-1}$. The chiral separation of $( \pm)$-GR24 was performed with a Chiralpak AD column, and each purified enantiomer was applied to the HPLC with a Chiralpak IA-3 column. We found that the compounds were eluted from a Chiralpak IA-3 column with the same order of elution as from a Chiralpak AD column. By comparing these data with the previously reported data using Chiralpak AD-H by Akiyama et al. ${ }^{8}$, it was confirmed that peak A represented ( - )-ent-GR24 (5) and peak B represented (+)-GR24 (2).

Trypsin digestion assay. Purified D14 protein, which was dissolved in a solution containing $20 \mathrm{mM}$ Tris- $\mathrm{HCl}(\mathrm{pH} 8.0)$ and $0.1 \%$ dimethylsulphoxide at the final concentration of $5 \mu \mathrm{M}$, was incubated in the absence or presence of $10 \mu \mathrm{M}( \pm)$ GR24 or $10 \mu \mathrm{M}(-)$-ent-2'-epi-GR7 (3) at $4^{\circ} \mathrm{C}$ for $15 \mathrm{~min}$. The D14 solution was mixed with a trypsin solution containing $1 \mathrm{mg} \mathrm{ml}^{-1}$ trypsin, $1 \mathrm{mM} \mathrm{HCl}$ and $2 \mathrm{mM} \mathrm{CaCl}_{2}$ by the volume ratio of $500: 3$ and was then incubated at $4{ }^{\circ} \mathrm{C}$ for
Table 1 | Data collection and refinement statistics.

\begin{tabular}{|c|c|c|}
\hline & D14 & D14 - D-OH \\
\hline \multicolumn{3}{|l|}{ Data collection* } \\
\hline X-ray source & $\begin{array}{c}\text { Photon Factory } \\
\text { AR-NE3A }\end{array}$ & $\begin{array}{l}\text { Rigaku FR-E rotating- } \\
\text { anode X-ray generator }\end{array}$ \\
\hline Wavelength $(\AA)$ & 1.0000 & 1.5418 \\
\hline Space group & $P 2_{1} 2_{1} 2_{1}$ & $P 2_{1} 2_{1} 2_{1}$ \\
\hline $\begin{array}{l}\text { Unit-cell parameters } \\
(\AA)\end{array}$ & $\begin{array}{c}a=48.1, b=88.7 \\
c=118.6\end{array}$ & $\begin{array}{c}a=48.4, b=88.1 \\
c=119.0\end{array}$ \\
\hline Resolution range $(\AA)$ & $20.0-1.75(1.80-1.75)$ & $20.0-2.10(2.15-2.10)$ \\
\hline $\begin{array}{l}\text { No. of observed } \\
\text { reflections }\end{array}$ & $37,0236(20,889)$ & $215,098(15,202)$ \\
\hline $\begin{array}{l}\text { No. of unique } \\
\text { reflections }\end{array}$ & $51,922(3790)$ & 30,394 (2169) \\
\hline Average redundancy & $7.1(5.5)$ & $7.1(7.0)$ \\
\hline Completeness (\%) & $99.8(99.2)$ & $99.8(100)$ \\
\hline$R_{\text {sym }}$ & $0.076(0.337)$ & $0.086(0.430)$ \\
\hline Average $I / \sigma(I)$ & $22.0(6.5)$ & $20.4(5.2)$ \\
\hline \multicolumn{3}{|l|}{ Refinement ${ }^{\star}$} \\
\hline$R_{\text {work }} / R_{\text {free }}(\%)$ & $18.3 / 22.3$ & $19.1 / 24.6$ \\
\hline No. of reflections & 49,279 & 28,858 \\
\hline \multicolumn{3}{|l|}{ No. of atoms } \\
\hline Protein & 4074 & 4108 \\
\hline Ligand & - & 8 \\
\hline Water & 340 & 195 \\
\hline \multicolumn{3}{|l|}{ Mean $B$-value $\left(\AA^{2}\right)$} \\
\hline Protein & 12.6 & 21.8 \\
\hline Ligand & - & 38.6 \\
\hline Water & 18.5 & 25.7 \\
\hline \multicolumn{3}{|l|}{ r.m.s.d. } \\
\hline Bond lengths $(\AA)$ & 0.023 & 0.022 \\
\hline Bond angles $\left({ }^{\circ}\right)$ & 1.933 & 1.879 \\
\hline
\end{tabular}

$0,1,2,4,8,12$ or $24 \mathrm{~h}$. The proteolysis reaction was stopped by mixing with $0.1 \mathrm{mM}$ phenylmethanesulfonyl fluoride and SDS-PAGE sample buffer. The digested proteins were analysed by SDS-PAGE and Coomassie Brilliant Blue G-250 stain.

Preparation of chemicals. GR24 and GR7 were prepared as described previously $^{53}$. SL analogues (compounds 10 - 13; Supplementary Fig. S4a) were prepared according to the previously described reaction ${ }^{54}$. Among them, we synthesized two novel compounds as follows.

4-methyl-5-oxo-2,5-dihydrofuran-2-yl 3,5-dimethoxybenzoate (3,5-dimethoxybenzoyloxy D-ring) (12). In a 50-ml flask with a magnetic stir bar, potassium carbonate $(0.18 \mathrm{~g}, 1.0 \mathrm{mmol})$ and tetra- $n$-butylammonium bromide $(0.32 \mathrm{~g}$, $1.0 \mathrm{mmol}$ ) were dissolved in a mixture of $\mathrm{CH}_{2} \mathrm{Cl}_{2}(2 \mathrm{ml})$ and $\mathrm{H}_{2} \mathrm{O}(3 \mathrm{ml})$. Into this solution, 3,5-dimethoxybenzoic acid $(0.35 \mathrm{~g}, 1.9 \mathrm{mmol})$ and 5-bromo-3-methyl$2(5 \mathrm{H})$-furanone $(0.17 \mathrm{~g}, 1.0 \mathrm{mmol})$ in $\mathrm{CH}_{2} \mathrm{Cl}_{2}(2 \mathrm{ml})$ were added and the mixture was stirred vigorously at room temperature for over $10 \mathrm{~h}$. The reaction mixture was added to $\mathrm{CH}_{2} \mathrm{Cl}_{2}$ and washed with brine, and the organic layer was dried over $\mathrm{Na}_{2} \mathrm{SO}_{4}$, and then filtered and concentrated in vacuo. The residue was purified by silica gel column chromatography and the resulting solid was recrystallized from ethanol/hexane to give 3,5-dimethoxybenzoyloxy D-ring (12) as a white solid. TLC (ethanol:hexane, 1:2 v/v): $R_{\mathrm{f}}=0.47 ;{ }^{1} \mathrm{H}$ NMR $\left(500 \mathrm{MHz}, \mathrm{CDCl}_{3}\right): \delta 7.17(2 \mathrm{H})$, $7.10(1 \mathrm{H}), 7.03(1 \mathrm{H}), 6.69(1 \mathrm{H}), 3.83(6 \mathrm{H}), 2.04(3 \mathrm{H}) .{ }^{13} \mathrm{C} \mathrm{NMR}\left(125.65 \mathrm{MHz} \mathrm{CDCl}_{3}\right)$ : $\delta 171.1(\mathrm{C} 1), 164.6(\mathrm{C} 1), 160.8(2 \mathrm{C}), 142.1(1 \mathrm{C}), 134.6(1 \mathrm{C}), 130.2(1 \mathrm{C}), 107.6(2 \mathrm{C})$, $106.8(1 \mathrm{C}), 93.2(1 \mathrm{C}), 55.6(2 \mathrm{C}), 10.6(1 \mathrm{C})$. HRMS $(\mathrm{m} / \mathrm{z}):[\mathrm{M}+\mathrm{H}]^{+}$calcd. for $\mathrm{C}_{14} \mathrm{H}_{15} \mathrm{O}_{6}, 279.0863$; found, 279.0874 .

4-methyl-5-oxo-2,5-dihydrofuran-2-yl anthracene-9-carboxylate (anthracene9-carbonyloxy D-ring) (13). In a 25-ml flask with a magnetic stir bar, sodium hydride $(60 \%$, dispersion in paraffin liquid, $0.05 \mathrm{~g}, 1.2 \mathrm{mmol})$, tetrahydrofuran (THF, $3 \mathrm{ml})$ and anthracene-9-carboxilic acid $(0.44 \mathrm{~g}, 2.0 \mathrm{mmol})$ in THF $(3 \mathrm{ml})$ were added and stirred. 5-bromo-3-methyl-2(5H)-furanone $(0.17 \mathrm{~g}, 1.0 \mathrm{mmol})$ in THF $(2 \mathrm{ml})$ was added and the mixture was stirred vigorously at room temperature for $4 \mathrm{~h}$. The reaction mixture was added to $\mathrm{CH}_{2} \mathrm{Cl}_{2}$ and washed with brine, and the organic layer was further extracted with brine twice. The combined aqueous layers were washed with ethanol twice and dried over $\mathrm{Na}_{2} \mathrm{SO}_{4}$, and then filtered and concentrated in vacuo. The residue was purified by silica gel column chromatography and the resulting solid was recrystallized from ethanol/hexane to give anthracene-9-carbonyloxy D-ring (13) as a yellow solid. TLC (ethanol:hexane, 
$1: 2 \mathrm{v} / \mathrm{v}): R_{\mathrm{f}}=0.48,{ }^{1} \mathrm{H}$ NMR $\left(500 \mathrm{MHz}, \mathrm{CDCl}_{3}\right): \delta 8.58(1 \mathrm{H}), 8.09(2 \mathrm{H}), 8.04(2 \mathrm{H})$, $7.59(2 \mathrm{H}), 7.51(2 \mathrm{H}), 7.46(1 \mathrm{H}), 7.08(1 \mathrm{H}), 2.01(3 \mathrm{H}) .{ }^{13} \mathrm{C}$ NMR $(125.65 \mathrm{MHz}$, $\left.\mathrm{CDCl}_{3}\right): \delta 171.0(1 \mathrm{C}), 167.8(1 \mathrm{C}), 141.8(1 \mathrm{C}), 134.9(1 \mathrm{C}), 130.8(2 \mathrm{C}), 130.5(2 \mathrm{C})$ 128.7 (3C), 127.6 (2C), 125.6 (2C), 125.1 (1C), 124.6 (2C), 93.0 (1C), 10.7 (1C).

\section{References}

1. Gomez-Roldan, V. et al. Strigolactone inhibition of shoot branching. Nature 455, 189-194 (2008).

2. Umehara, M. et al. Inhibition of shoot branching by new terpenoid plant hormones. Nature 455, 195-200 (2008).

3. Cook, C. E., Whichard, L. P., Turner, B., Wall, M. E. \& Egley, G. H. Germination of Witchweed (Striga lutea Lour.): isolation and properties of a potent stimulant. Science 154, 1189-1190 (1966).

4. Yoneyama, K., Awad, A. A., Xie, X. \& Takeuchi, Y. Strigolactones as germination stimulants for root parasitic plants. Plant Cell Physiol. 51, 1095-1103 (2010)

5. Parker, C. Observations on the current status of Orobanche and Striga problems worldwide. Pest Manag. Sci. 65, 453-459 (2009).

6. Akiyama, K., Matsuzaki, K. \& Hayashi, H. Plant sesquiterpenes induce hyphal branching in arbuscular mycorrhizal fungi. Nature 435, 824-827 (2005)

7. Akiyama, K. \& Hayashi, H. Strigolactones: chemical signals for fungal symbionts and parasitic weeds in plant roots. Ann. Bot. 97, 925-931 (2006)

8. Akiyama, K., Ogasawara, S., Ito, S. \& Hayashi, H. Structural requirements of strigolactones for hyphal branching in AM fungi. Plant Cell Physiol. 51, 1104-1117 (2010).

9. Alder, A. et al. The path from $\beta$-carotene to carlactone, a strigolactone-like plant hormone. Science 335, 1348-1351 (2012).

10. Beveridge, C. A. Axillary bud outgrowth: sending a message. Curr. Opin. Plant Biol. 9, 35-40 (2006).

11. Ongaro, V. \& Leyser, O. Hormonal control of shoot branching. J. Exp. Bot. 59, 67-74 (2008).

12. Wang, Y. \& Li, J. Branching in rice. Curr. Opin. Plant Biol. 14, 94-99 (2011).

13. Arite, T. et al. d14, a strigolactone-insensitive mutant of rice, shows an accelerated outgrowth of tillers. Plant Cell Physiol. 50, 1416-1424 (2009).

14. Waters, M. T. et al. Specialisation within the DWARF14 protein family confers distinct responses to karrikins and strigolactones in Arabidopsis. Development 139, 1285-1295 (2012).

15. Gao, Z. et al. Dwarf88, a novel putative esterase gene affecting architecture of rice plant. Plant Mol. Biol. 71, 265-276 (2009).

16. Liu, W. et al. Identification and characterization of HTD2: a novel gene negatively regulating tiller bud outgrowth in rice. Planta 230, 649-658 (2009).

17. Stirnberg, P., van De Sande, K. \& Leyser, H. M. MAX1 and MAX2 control shoot lateral branching in Arabidopsis. Development 129, 1131-1141 (2002).

18. Stirnberg, P., Furner, I. J. \& Ottoline Leyser, H. M. MAX2 participates in an SCF complex which acts locally at the node to suppress shoot branching. Plant J. 50, 80-94 (2007).

19. Ishikawa, S. et al. Suppression of tiller bud activity in tillering dwarf mutants of rice. Plant Cell Physiol. 46, 79-86 (2005).

20. Johnson, X. et al. Branching genes are conserved across species. Genes controlling a novel signal in pea are coregulated by other long-distance signals. Plant Physiol. 142, 1014-1026 (2006).

21. Woo, H. R. et al. ORE9, an F-box protein that regulates leaf senescence in Arabidopsis. Plant Cell 13, 1779-1790 (2001).

22. Shen, H., Luong, P. \& Huq, E. The F-box protein MAX2 functions as a positive regulator of photomorphogenesis in Arabidopsis. Plant Physiol. 145, 1471-1483 (2007)

23. Tsuchiya, Y. et al. A small-molecule screen identifies new functions for the plant hormone strigolactone. Nat. Chem. Biol. 6, 741-749 (2010).

24. Toh, S. et al. Thermoinhibition uncovers a role for strigolactones in Arabidopsis seed germination. Plant Cell Physiol. 53, 107-117 (2012).

25. Shen, H., Zhu, L., Bu, Q. Y. \& Huq, E. MAX2 affects multiple hormones to promote photomorphogenesis. Mol. Plant 5, 224-236 (2012).

26. $\mathrm{Hu}, \mathrm{Z}$. et al. Strigolactones negatively regulate mesocotyl elongation in rice during germination and growth in darkness. Plant Cell Physiol. 51, 1136-1142 (2010).

27. Nelson, D. C. et al. F-box protein MAX2 has dual roles in karrikin and strigolactone signaling in Arabidopsis thaliana. Proc. Natl Acad. Sci. USA 108, 8897-8902 (2011).

28. Scaffidi, A. et al. Exploring the molecular mechanism of karrikins and strigolactones. Bioorg. Med. Chem. Lett. 22, 3743-3746 (2012).

29. Kapulnik, Y. et al. Strigolactones affect lateral root formation and root-hair elongation in Arabidopsis. Planta 233, 209-216 (2011).

30. Rasmussen, A. et al. Strigolactones suppress adventitious rooting in Arabidopsis and pea. Plant Physiol. 158, 1976-1987 (2012).

31. Hamiaux, C. et al. DAD2 is an $\alpha / \beta$ hydrolase likely to be involved in the perception of the plant branching hormone, strigolactone. Curr. Biol. 22, 2032-2036 (2012)
32. Lo, S. F. et al. A novel class of gibberellin 2-oxidases control semidwarfism, tillering, and root development in rice. Plant Cell 20, 2603-2618 (2008).

33. Hou, X. et al. DELLAs modulate jasmonate signaling via competitive binding to JAZs. Dev. Cell 14, 884-894 (2010).

34. Yang, D. L. et al. Plant hormone jasmonate prioritizes defense over growth by interfering with gibberellin signaling cascade. Proc. Natl Acad. Sci. USA 109, E1192-E1200 (2012)

35. Gallego-Bartolomé, J. et al. Molecular mechanism for the interaction between gibberellin and brassinosteroid signaling pathways in Arabidopsis. Proc. Natl Acad. Sci. USA 109, 13446-13451 (2012).

36. Bai, M. Y. et al. Brassinosteroid, gibberellin and phytochrome impinge on a common transcription module in Arabidopsis. Nat. Cell Biol. 14, 810-817 (2012).

37. Wang, F. \& Deng, X. W. Plant ubiquitin-proteasome pathway and its role in gibberellin signaling. Cell Res. 21, 1286-1294 (2011).

38. Ueguchi-Tanaka, M. et al. gibberellin insensitive dwarf1 encodes a soluble receptor for gibberellin. Nature 437, 693-698 (2005).

39. Silverstone, A. L., Ciampaglio, C. N. \& Sun, T. The Arabidopsis RGA gene encodes a transcriptional regulator repressing the gibberellin signal transduction pathway. Plant Cell 10, 155-169 (1998).

40. Kagiyama, M. et al. Structures of D14 and D14L in the strigolactone and karrikin signaling pathways. Genes Cells 18, 147-160 (2013).

41. Zhao, L. H. et al. Crystal structures of two phytohormone signal-transducing $\alpha / \beta$ hydrolases: karrikin-signaling KAI2 and strigolactone-signaling DWARF14. Cell Res. 23, 436-439 (2013).

42. Boyer, F. D. et al. Structure-activity relationship studies of strigolactone-related molecules for branching inhibition in garden pea: molecule design for shoot branching. Plant Physiol. 159, 1524-1544 (2012).

43. Ito, S. et al. A new lead chemical for strigolactone biosynthesis inhibitors. Plant Cell Physiol. 51, 1143-1150 (2010).

44. Nakagawa, T. et al. Development of series of gateway binary vectors, pGWBs, for realizing efficient construction of fusion genes for plant transformation. J. Biosci. Bioeng. 104, 34-41 (2007).

45. Ueda, T., Yamaguchi, M., Uchimiya, H. \& Nakano, A. Ara6, a plant-unique novel type Rab GTPase, functions in the endocytic pathway of Arabidopsis thaliana. EMBO J. 20, 4730-4741 (2001).

46. Goh, T. et al. VPS9a, the common activator for two distinct types of Rab5 GTPases, is essential for the development of Arabidopsis thaliana. Plant Cell 19, 3504-3515 (2007).

47. Ito, E. et al. Dynamic behavior of clathrin in Arabidopsis thaliana unveiled by live imaging. Plant J. 69, 204-216 (2012)

48. Kabsch, W. Automatic processing of rotation diffraction data from crystals of initially unknown symmetry and cell constants. J. Appl. Cryst. 26, 795-800 (1993).

49. Kaneko, T., Tanaka, N. \& Kumasaka, T. Crystal structures of RsbQ, a stressresponse regulator in Bacillus subtilis. Protein Sci. 14, 558-565 (2005).

50. Emsley, P. \& Cowtan, K. Coot: model-building tools for molecular graphics. Acta Crystallogr. D Biol. Crystallogr. 60, 2126-2132 (2004).

51. Murshudov, G. N., Vagin, A. A. \& Dodson, E. J. Refinement of macromolecular structures by the maximum-likelihood method. Acta Crystallogr. D Biol. Crystallogr. 53, 240-255 (1997).

52. Laskowski, R. A., MacArthur, M. W., Moss, D. S. \& Thornson, J. M. PROCHECK: a program to check the stereochemical quality of protein structures. J. Appl. Cryst. 26, 283-391 (1993).

53. Mangnus, E., Dommerholt, F., Dejong, R. \& Zwanenburg, B. Improved synthesis of strigol analog GR24 and evaluation of the biological activity of its diastereomers. J. Agric. Food Chem. 40, 1230-1235 (1992).

54. Fukui, K. et al. New branching inhibitors and their potential as strigolactone mimics in rice. Bioorg. Med. Chem. Lett. 21, 4905-4908 (2011).

\section{Acknowledgements}

We thank J. Kyozuka (The University of Tokyo) for providing rice cultivar Shiokari and d10-1 and $d 27-1$ mutants, and T.Nakagawa (Shimane University) for providing the pUGW vectors. This work was supported in part with grants from the Program for Promotion of Basic Research Activities for Innovative Biosciences (PROBRAIN), the Japan Society for the Promotion of Science (JSPS) Grant-in-Aid for Scientific Research (A), and the Core Research for Evolutional Science and Technology (CREST) Program of Japan Science and Technology Agency (JST) (T.A.), and the Targeted Proteins Research Program (TPRP) from the Ministry of Education, Culture, Sports, Science, and Technology of Japan (MEXT) (M.T.). The synchrotron-radiation experiments were performed at AR-NE3A in the Photon Factory (Tsukuba) (2008S2-001).

\section{Authors contributions}

T.A. and M.T. conceived and designed the project. H.N. performed the Y2H experiments. S.I., K.F. and N.T. prepared the SL compounds. S.I., N.T. and H.N. performed the branching inhibition assay in rice. H.N., E.I. and T.U. performed the BiFC experiments. S.-H.P. performed the Y3H assays. Y.-L.X., T.M., F.H. and H.-M. Q. performed the construct design, subcloning, protein expression and purification. H.N. performed the SL-binding assay. Y.-L.X. crystallized D14 and its complex with a ligand. Y.-L.X. and 
T.M. collected the X-ray diffraction data and analysed the protein structures. H.N. and K.F. performed the enzymatic GR24-degradation experiments of D14 and the HPLC analyses. X.S., Y.M. and A.A. performed the protein expression and purification. H.N., Y.-L.X. and T.M. wrote the manuscript. F.H. assisted in the preparation of the manuscript and figures. T.A. and M.T. edited the manuscript.

\section{Additional information}

Accession codes. The structure coordinates and structural factors have been deposited in the Protein Data Bank under accession codes 3VXK (D14) and 3WIO (D14 - D-OH complex).
Supplementary Information accompanies this paper at http://www.nature.com/ naturecommunications

Competing financial interests: The authors declare no competing financial interests.

Reprints and permission information is available online at http://npg.nature.com/ reprintsandpermissions/

How to cite this article: Nakamura, H. et al. Molecular mechanism of strigolactone perception by DWARF14. Nat. Commun. 4:2613 doi: 10.1038/ncomms3613 (2013). 